\title{
RANDOM REARRANGEMENTS OF FOURIER COEFFICIENTS
}

BY DANIEL M. OBERLIN

Communicated by R. R. Goldberg, February 3, 1976

In [2] and [3] Hardy and Littlewood characterized those sequences of numbers which for every variation of their arguments and arrangements are the Fourier coefficients of a function in $L^{p}(2<p<\infty)$ and those sequences which for some variation of their arguments and arrangements are the Fourier coefficients of a function in $L^{p}(1<p<2)$. In [4] Paley and Zygmund characterized those sequences $\left(c_{n}\right)$ such that for almost every choice of \pm 1 's, $\left( \pm c_{n}\right)$ is the sequence of Fourier coefficients of a function in $L^{p}(1 \leqslant p<\infty)$. Here we are interested in the following problem: Which sequences are for almost every variation of their arrangements the sequence of Fourier coefficients of a function in $L^{p}$ ? Of course it is necessary to make precise the phrase "almost every variation of their arrangements". Following Garsia [1] we consider a probability space $X$ of "local" permutations of $\{1,2, \ldots\}$ : For $k=0,1,2, \ldots$ let $S\left(2^{k}\right)$ be the symmetric group on the set $\left\{2^{k}, 2^{k}+1, \ldots, 2^{k+1}-1\right\}$. To each $\sigma_{k} \in S\left(2^{k}\right)$ we assign the probability $1 / 2^{k}$, and we let $X$ be the product probability space $\Pi_{k=0}^{\infty} S\left(2^{k}\right)$. For $\sigma=\left(\sigma_{0}, \sigma_{1}, \ldots\right) \in X$ and a Fourier series of the form $\Sigma_{n=1}^{\infty} c_{n} e^{i n \theta}$, we define

$$
S(\sigma, \theta)=\sum_{k=0}^{\infty} \sum_{2^{k} \leqslant n<2} c_{\sigma_{k}(n)} e^{i n \theta} .
$$

For $k=0,1, \ldots$ let

$$
a_{k}=2^{-k} \sum_{2^{k \leqslant n<2} k+1} c_{n},
$$

and let $(*)$ stand for the statement that

$$
\sum_{k=0}^{\infty}\left|a_{k}\right|^{p} 2^{k(p-1)}<\infty \text { and } \sum_{k=0}^{\infty} \sum_{2^{k} \leqslant n<2 k+1}\left|c_{n}-a_{k}\right|^{2}<\infty .
$$

THEOREM 1. For $2<p<\infty$, the following are equivalent:

(i) for some $\sigma \in X, S(\sigma, \theta)$ is an $L^{p}$ Fourier series;

(ii) $S(\sigma, \theta)$ is almost surely an $L^{p}$ Fourier series;

(iii) $(*)$.

THEOREM 2. For $1<p<2$, the following are equivalent:

(i) $S(\sigma, \theta)$ is an $L^{p}$ Fourier series with positive probability;

AMS (MOS) subject classifications (1970). Primary 42 A36.

Copyright () 1976, American Mathematical Society 
(ii) $S(\sigma, \theta)$ is an $L^{p}$ Fourier series for every $\sigma \in X$;

(iii) $(*)$.

THEOREM 3. If $a_{k}=0$ for $k=0,1,2, \ldots$, the following are equivalent:

(i) $S(\sigma, \theta)$ is a Fourier (-Stieltjes) series with positive probability;

(ii) it is almost sure that $S(\sigma, \theta)$ is the Fourier series of a function in every $L^{p}(1 \leqslant p<\infty)$.

The proofs are based on a combinatorial estimation of the $L^{p}$ norms of permuted Fourier series on finite groups and on classical results due to Hardy, Littlewood, Paley, and others. Analogous results obtain for Fourier series on the group $D^{\infty}=\Pi_{n=1}^{\infty}\{0,1\}$.

\section{REFERENCES}

1. A. M. Garsia, Existence of almost everywhere convergent rearrangements for Fourier series of $L_{2}$ functions, Ann. of Math. (2) 79 (1964), 623-629. MR 28 \#4288.

2. G. H. Hardy and J. E. Littlewood, Some new properties of Fourier constants, Math. Ann. 97 (1926), 159-209.

3. - Some new properties of Fourier constants, J. London Math. Soc. 6 (1931), 3-9.

4. R. E. A. C. Paley and A. Zygmund, On some series of functions. I, II, III, Proc. Cambridge Philos. Soc. 26 (1930), 337-357, 458-474; ibid, 28 (1932), 190-205.

DEPARTMENT OF MATHEMATICS, FLORIDA STATE UNIVERSITY, TALLAHASSEE, FLORIDA 32306 